\title{
Prognostic role of lemur tyrosine kinase 3 in postoperative gastric cancer
}

\author{
ZHENGGUANG LI, JUN WU, MEI JI, LIANGRONG SHI, BIN XU, JINGTING JIANG and CHANGPING WU \\ Department of Oncology, The Third Affiliated Hospital of Soochow University, Changzhou, Jiangsu 213003, P.R. China
}

Received January 23, 2014; Accepted March 18, 2014

DOI: $10.3892 / \mathrm{mco} .2014 .301$

\begin{abstract}
The treatment of gastric cancer has been unsatisfactory thus far; therefore, novel targets and treatment strategies are urgently required. Lemur tyrosine kinase (LMTK) 3 is an estrogen receptor- $\alpha(\mathrm{ER} \alpha)$ modulator with a central role in endocrine resistance in breast cancer. Moreover, the expression and polymorphisms of LMTK3 are correlated with the prognosis of breast cancer patients. Since estrogen receptor (ER) is also expressed and plays a role in gastric cancer, we herein investigated the expression of the LMTK3 protein in 83 gastric cancer patients by tissue microarray and analyzed the correlation between LMTK3 expression and the prognosis of gastric cancer. Our results demonstrated that LMTK3 was more frequently expressed in gastric cancer tissues compared to non-cancerous mucosa (79.5 vs. $45.8 \%$, respectively; $\mathrm{P}=0.000$ ). The LMTK3 expression was significantly correlated with the depth of invasion $(\mathrm{P}=0.002)$ and disease stage $(\mathrm{P}=0.035)$. The Kaplan-Meier analysis revealed that the postoperative survival of the LMTK3-negative group was superior to that of the LMTK3-positive group $(\mathrm{P}=0.043)$. Moreover, the multivariate analysis identified LMTK3 expression as an independent prognostic factor for patients with gastric cancer $(\mathrm{P}=0.019)$. These findings suggested that the expression of LMTK3 may be a negative prognostic factor in patients with gastric cancer. Moreover, targeting LMTK3 is a potential strategy for the treatment of gastric cancer, although the biological functions of LMTK3 in gastric cancer require further investigation.
\end{abstract}

\section{Introduction}

The incidence of gastric cancer has been on the decline globally over the last few years, although it remains rampant in several countries and is currently the fourth most common type of cancer worldwide $(1,2)$. In China, in particular, more new cases

Correspondence to: Professor Changping Wu, Department of Oncology, The Third Affiliated Hospital of Soochow University, 185 Juqian Street, Changzhou, Jiangsu 213003, P.R. China

E-mail: wcpzlk@163.com

Key words: lemur tyrosine kinase-3, gastric cancer, prognosis, endocrine therapy of gastric cancer are diagnosed annually compared to any other country. Gastric cancer is difficult to diagnose in an early stage and the long-term prognosis remains disappointing $(3,4)$. Over the last few decades, there have been no notable advances in conventional therapy and the median survival of patients with advanced gastric cancer ranges between 9 and 11 months. Targeted therapy is a novel promising strategy for the treatment of malignancies. In gastric cancer, trastuzumab was shown to reduce the risk of death by $26 \%$ in the ToGA trial, when combined with chemotherapy in patients exhibiting high human epidermal growth factor receptor 2 (HER2) expression (5). However, the median overall survival in that study was only 13.8 months and the results were unsatisfactory. Moreover, a series of clinical trials indicated that there is currently no molecular-targeted agent superior to trastuzumab (6). Therefore, further investigation is required to elucidate the molecular mechanisms underlying the development of gastric cancer, identify novel treatment targets or design therapeutic strategies.

Lemur tyrosine kinase (LMTK)3, which is a member of the LMTK family, is a serine/threonine-protein kinase that may be involved in the $\beta$-catenin pathway and leukemic cell survival $(7,8)$; however, the biological function of the gene remains unclear. Giamas et al (9) recently demonstrated that LMTK3 is an estrogen receptor- $\alpha(E R \alpha)$ regulator, with a central role in endocrine resistance. Moreover, the expression of LMTK3 was associated with breast cancer phenotype and patient prognosis (10).

It was reported that estrogens may play a role in gastric carcinogenesis (11) and tamoxifen may prevent gastric cancer in Helicobacter pylori-infected INS-GAS mice (12). Furthermore, estrogen receptor (ER) has been detected in gastric cancer tissues and proven to be correlated with prognosis (13-15). Therefore, ER and related genes may represent potential targets for the treatment of gastric cancer (16). In view of the role of LMTK3 in breast cancer, we herein investigated the expression of LMTK3 and its correlation with the clinicopathological parameters and prognosis of gastric cancer patients.

\section{Materials and methods}

Materials. The gastric cancer tissue microarray (TMA) was purchased form Outdo Biotech Co., Shanghai, China. The tumor samples were obtained from 83 patients with primary operable gastric cancer who had undergone surgical resection between 2006 and 2007. All the patients in this series were 
Table I. Association between LMTK3 expression and clinicopathological characteristics in gastric cancer.

\begin{tabular}{|c|c|c|c|c|c|}
\hline \multirow[b]{2}{*}{ Characteristics } & \multirow[b]{2}{*}{ Cases } & \multicolumn{2}{|c|}{ LMTK3 } & \multirow[b]{2}{*}{$\chi^{2}$} & \multirow[b]{2}{*}{ P-value } \\
\hline & & Negative & Positive (\%) & & \\
\hline Tissue & & & & 25.739 & 0.000 \\
\hline Gastric cancer & 83 & 17 & $66(79.5)$ & & \\
\hline Non-cancerous mucosa & 83 & 45 & $38(45.8)$ & & \\
\hline Age (years) & & & & 0.004 & 0.948 \\
\hline$\leq 50$ & 7 & 2 & $5(6.0)$ & & \\
\hline$>50$ & 76 & 15 & $61(73.5)$ & & \\
\hline Gender & & & & 0.996 & 0.318 \\
\hline Male & 55 & 13 & $42(50.6)$ & & \\
\hline Female & 28 & 4 & $24(28.9)$ & & \\
\hline Stage & & & & 4.445 & 0.035 \\
\hline I+II & & 11 & $20(24.1)$ & & \\
\hline $\mathrm{III}+\mathrm{IV}$ & & 8 & $44(53.0)$ & & \\
\hline Tumor size (cm) & & & & 0.284 & 0.594 \\
\hline$\leq 5$ & 49 & 11 & $38(45.8)$ & & \\
\hline$>5$ & 34 & 6 & $28(33.7)$ & & \\
\hline Depth of invasion & & & & 9.272 & 0.002 \\
\hline $\mathrm{T} 1+\mathrm{T} 2$ & 17 & 8 & $9(10.8)$ & & \\
\hline $\mathrm{T} 3+\mathrm{T} 4$ & 66 & 9 & $57(68.7)$ & & \\
\hline Lymph node metastasis & & & & 0.328 & 0.567 \\
\hline $\mathrm{N} 0+\mathrm{N} 1$ & & 8 & $26(31.3)$ & & \\
\hline $\mathrm{N} 2+\mathrm{N} 3$ & & 9 & $40(48.2)$ & & \\
\hline Distant metastasis & & & & 1.381 & 0.240 \\
\hline M0 & 74 & 17 & $57(68.7)$ & & \\
\hline M1 & 9 & 0 & $9(10.8)$ & & \\
\hline Lymphatic and vascular invasion & & & & 0.987 & 0.321 \\
\hline Negative & 69 & 16 & $53(63.9)$ & & \\
\hline Positive & 14 & 1 & $13(15.7)$ & & \\
\hline Location & & & & 0.125 & 0.940 \\
\hline Upper & 8 & 2 & $6(7.2)$ & & \\
\hline Middle & 29 & 6 & $23(27.7)$ & & \\
\hline Lower & 46 & 9 & $37(44.6)$ & & \\
\hline Grade & & & & 1.99 & 0.158 \\
\hline $\mathrm{I}+\mathrm{II}$ & 37 & 5 & $32(38.6)$ & & \\
\hline III+IV & 46 & 12 & $34(41.0)$ & & \\
\hline
\end{tabular}

LMTK3, lemur tyrosine kinase 3.

Chinese, including 55 men and 28 women, with a mean age of 64.3 years (range, $37-84$ years). All the cases were evaluable after array construction. All the patients underwent potentially curative tumor resection and none had received chemotherapy or radiotherapy prior to surgery. The tumor histological types and grading were reviewed and classified according to the WHO classification criteria; the disease stage was determined according to the TNM staging system.

Immunohistochemistry. Anti-LMTK3 mouse monoclonal antibody (Santa Cruz Biotechnology, Inc., Heidelberg, Germany) was optimized to a working concentration of $2 \mu \mathrm{g} / \mathrm{ml}$ on full-face excisional tissue sections. Subsequently, gastric cancer TMA was conducted, comprising 4- $\mu \mathrm{m}$ formalin-fixed paraffin-embedded tissue cores immunostained with the optimized anti-LMTK3 monoclonal antibody on the Leica BOND-MAX automated system (Leica Microsystems Inc., Buffalo Grove, IL, USA) according to the manufacturer's instructions. Heat-induced epitope retrieval was performed in citrate buffer (ER1) for $5 \mathrm{~min}$. Detection was achieved using the Polymer Detection kit (Leica Microsystems Inc., Newcastle Upon Tyne, UK). These detection systems contain 
peroxidase block, protein block, post primary block, Novolink polymer, 3,3'-diaminobenzidine (DAB) chromogen, Novolink DAB substrate buffer (polymer) and hematoxylin for subsequent counterstaining of the TMAs. Negative controls were performed by omission of the primary antibody.

LMTK3 immunoreactivity was detected in the nucleus and cytoplasm of gastric cancer cells to a variable degree. The stained specimens were then categorized into 6-degree classes according to the quantitative score. Initially, 4 degrees of the proportional score (PS) for the positively-stained cells were assigned as follows, according to the frequency of positive tumor cells: 0 , none; $1,1 / 100-1 / 4 ; 2,1 / 4-1 / 2$; and $3,>1 / 2$. Thereafter, 4 degrees of the intensity score (IS) were assigned as follows, according to the intensity of the staining: 0 , none; 1 , weak; 2 , intermediate; and 3 , strong. The PS and the IS were then added to obtain a total score (TS), which ranged between 0 and 6 . According to the TS, the LMTK3 expression of the tumor was classified as negative when the score was 0-3 and as positive when the score was 4-6. All the cases were independently scored by two of the investigators who were blinded to the clinicopathological or outcome data. In case of discrepancies between the two investigators, a consensus was reached via simultaneous examination using a double-headed microscope.

Statistics. The statistical analysis for TMAs was performed using SPSS version 16.0 statistical software (SPSS Inc., Chicago, IL, USA). The statistical significance was evaluated using the Chi-square test or the Fisher's exact test. A multivariate COX regression analysis was used to evaluate independent associations. The survival curves were analyzed using the Kaplan-Meier method and the significance was determined by the log-rank test. The odds ratio and $95 \%$ confidence interval (95\% CI) was calculated for each variable. $\mathrm{P}<0.05$ was considered to indicate statistically significant differences.

\section{Results}

Detection of the LMTK3 expression in gastric cancer. Immunostaining analysis revealed that LMTK3 was detected predominantly in the nucleus of gastric cancer cells, with variable cytoplasmic staining (Fig. 1). LMTK3 was also expressed in the adjacent non-cancerous mucosa; however, the positive rate in gastric cancer tissue was significantly higher compared to that in non-cancerous mucosa (79.5 vs. $45.8 \%$, respectively; $\mathrm{P}=0.000$; Table I).

Correlation of LMTK3 expression with clinicopathological parameters and postoperative survival in gastric cancer. In the present study, we investigated the correlation of LMTK3 expression with clinicopathological parameters and postoperative survival. Our results suggested that LMTK3 expression was significantly correlated with the depth of invasion $(\mathrm{P}=0.002)$ and disease stage $(\mathrm{P}=0.035)$, which were obviously associated with the prognosis of gastric cancer patients. There was no significant correlation with the other clinicopathological parameters (Table I).

The Kaplan-Meier analysis revealed that the postoperative survival of the LMTK3-negative group tended to be superior to that of the LMTK3-positive group, with a statistically significant difference ( $\mathrm{P}=0.043$; Fig. 2). Furthermore, as
Table II. Multivariate survival analysis of prognostic factors by Cox regression analysis.

\begin{tabular}{lcc}
$\begin{array}{l}\text { Clinicopathological } \\
\text { parameters }\end{array}$ & HR $(95 \%$ CI $)$ & P-value \\
\hline $\begin{array}{l}\text { LMTK3 } \\
\text { Higher/lower }\end{array}$ & $3.071(1.375-9.283)$ & 0.019 \\
$\begin{array}{l}\text { Gender } \\
\text { Female/male }\end{array}$ & $0.684(0.375-1.247)$ & 0.215 \\
$\begin{array}{l}\text { Age }(\text { years }) \\
\quad 50 />50\end{array}$ & $0.896(0.350-2.295)$ & 0.819 \\
$\begin{array}{l}\text { Grade } \\
\text { III+IV/I+II }\end{array}$ & $1.373(0.881-2.139)$ & 0.161 \\
$\begin{array}{l}\text { Size }(\mathrm{cm}) \\
>5 / \leq 5\end{array}$ & $1.279(0.720-2.727)$ & 0.401 \\
$\begin{array}{l}\text { TNM stage } \\
\text { III+IV/I+II }\end{array}$ & $3.642(2.934-10.887)$ & 0.015
\end{tabular}

HR, hazard risk; CI, confidence interval; LMTK3, lemur tyrosine kinase 3 .

shown in Table II, the multivariate analysis confirmed that the hazard risk (HR) of death was significantly higher in the group with positive LMTK3 staining compared to the negative group (HR=3.071, 95\% CI: 1.375-9.283). Therefore, it was suggested that positive LMTK3 staining may be associated with a poorer prognosis in patients with gastric cancer.

\section{Discussion}

Gastric cancer is the second leading cause of cancer-related mortality worldwide; however, over the last few decades, there have been no significant advances in the treatment of gastric cancer and the conventionally used methods have been proven unsatisfactory. Molecular-targeted therapy has become indispensable in the treatment of malignancies, such as trastuzumab for breast cancer and gefitinib or erlotinib for non-small-cell lung cancer. However, to date, only a limited number of targeted agents were shown to be of clinical benefit for gastric cancer, including trastuzumab and ramucirumab (17). Due to the significant heterogeneity of gastric cancer, HER2 is only overexpressed in only a few patients and the majority of the patients cannot benefit from trastuzumab $(18,19)$. To overcome the difficulties in gastric cancer treatment, more efficient biomarkers, molecular targets and novel therapeutic strategies are urgently required.

Endocrine therapy is important for the treatment of hormone-dependent malignancies, such as breast and prostate cancer. Estrogen and ER also play and important role in gastric cancer. According to epidemiological evidence and animal studies, estrogen plays a protective role in gastric tumorigenesis (20-22). Although not a direct target organ of sex hormones, ER $\alpha$ and $E R \beta$ were both found to be expressed in gastric cancer tissue. Further investigation demonstrated that the expression of ER was correlated with the prognosis of gastric cancer patients, with the two subtypes playing 

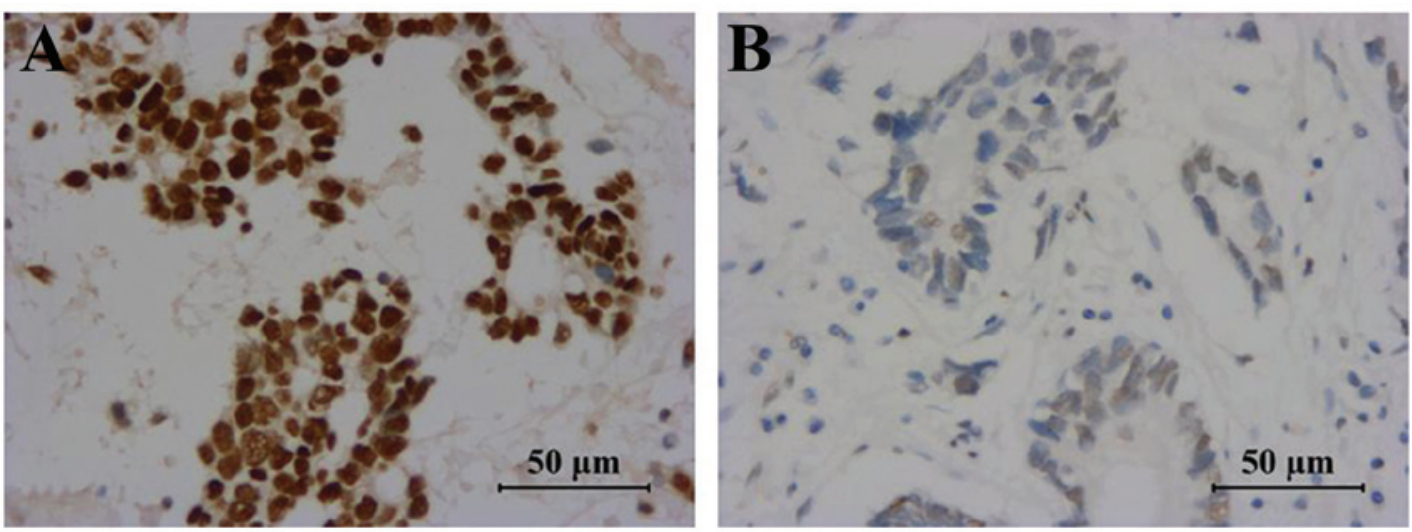

Figure 1. Immunohistochemical analysis of lemur tyrosine kinase (LMTK) 3 expression with anti-LMTK3 mouse monoclonal antibody in gastric cancer (magnification, x400). (A) LMTK3-positive staining; and (B) LMTK3-negative staining.

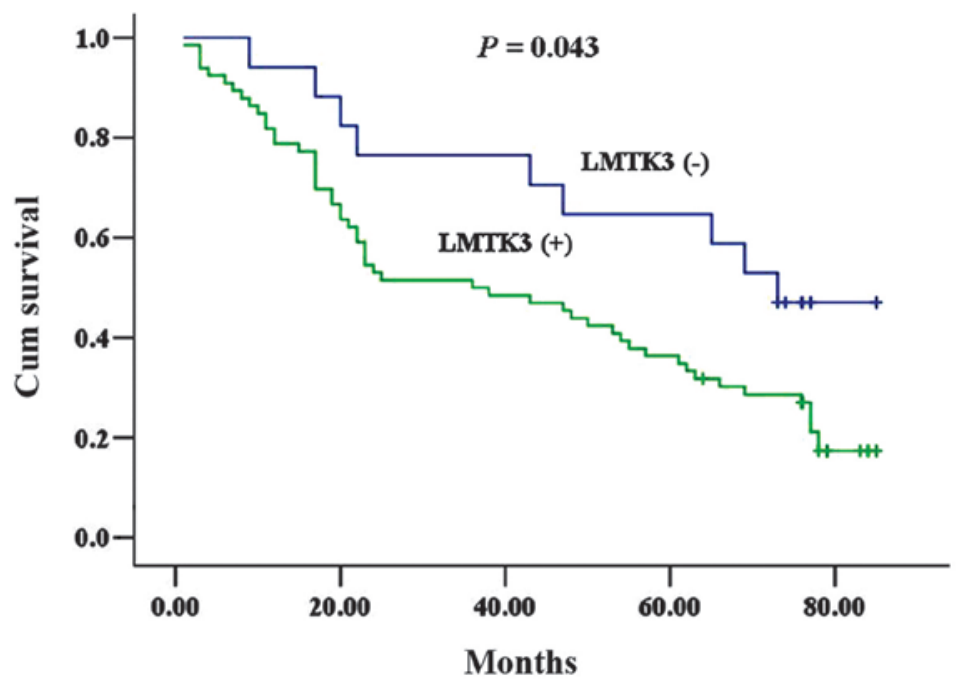

Figure 2. Kaplan-Meier survival curve in patients with gastric cancer for lemur tyrosine kinase (LMTK) 3 expression. Patients with positive LMTK3 expression (blue line) exhibited a tendency toward decreased postoperative survival compared to patients with negative LMTK3 expression (green line), with a statistically significant difference $(\mathrm{P}=0.043)$.

different roles (13,14,23-26). As regards ER, certain studies on anti-estrogen endocrine therapy have been conducted on gastric cancer; however, the results were not satisfactory and did not significantly affect the treatment outcome (27-30). Despite the unsatisfactory attempts, it was suggested that endocrine therapy may be a useful strategy for the treatment of gastric cancer, provided another efficient related target was utilized to improve the effect, rather than directly blocking ER (31).

The first studies on LMTK3 were mainly focused on breast cancer. However, we recently demonstrated the presence of LMTK3 protein in the blood of patients with colorectal cancer and serum LMTK3 may be a valuable biomarker for predicting disease progression and prognosis in such patients (32). Wakatsuki et al (33) reported that LMTK3 polymorphisms are correlated with the prognosis of gastric cancer, but they did not extensively investigate LMTK3 protein expression. In the present study, we demonstrated that the positive rate of LMTK3 protein expression in gastric cancer was significantly higher compared to that in the adjacent non-cancerous mucosa. Furthermore, the expression of LMTK3 was correlated with depth of invasion and cancer stage. In addition, LMTK3 appeared to be strongly positive in all 9 specimens with distant metastasis (Table I), strongly indicating that the expression of LMTK3 is correlated with metastasis of gastric cancer, if the sample is of adequate size. Furthermore, the survival analysis demonstrated that the expression of LMTK3 was negatively associated with postoperative survival in gastric cancer patients. The multivariate analysis identified the expression of LMTK3 as an independent negative prognostic factor in gastric cancer.

In breast cancer, LMTK3 was shown to induce the estrogen pathway via ER $\alpha$ phosphorylation and high baseline LMTK3 expression was found to be associated with a poorer overall and disease-free survival $(9,10)$. Of note, LMTK3 silencing through RNA interference significantly enhanced the growth inhibitory effect of tamoxifen in tamoxifen-resistant breast cancer cell lines (9). According to the function of LMTK3 in breast cancer, targeted inhibition of LMTK3 aimed at enhancing the effect of endocrine therapy may be an efficient treatment strategy for gastric cancer, particularly in patients expressing ER $\alpha$. 
In conclusion, our results demonstrated that the expression of LMTK3 may be a useful biomarker as a negative prognostic factor in gastric cancer and a potential novel target for the treatment of gastric cancer. Given the differences in the ER subtype distribution between breast and gastric cancer, LMTK3 may be more than a modulator of ER $\alpha$ in gastric cancer (33). The association between LMTK3 and ER $\beta$ requires further investigation and the role of LMTK3 in the development of gastric cancer should be elucidated by further studies.

\section{Acknowledgements}

This study was supported by grants from the Technology Project of Changzhou Social Development (no. CS20102016) and the Natural Science Funds for Young Teacher of Soochow University (no. Q3124943).

\section{References}

1. Siegel R, Naishadham D and Jemal A: Cancer statistics, 2012. CA Cancer J Clin 62: 10-29, 2012.

2. Kamangar F, Dores GM and Anderson WF: Patterns of cancer incidence, mortality, and prevalence across five continents: defining priorities to reduce cancer disparities in different geographic regions of the world. J Clin Oncol 24: 2137-2150, 2006.

3. Huang JY, Xu YY, Sun Z, et al: Comparison different methods of intraoperative and intraperitoneal chemotherapy for patients with gastric cancer: a meta-analysis. Asian Pac J Cancer Prev 13 4379-4385, 2012.

4. Meyer HJ and Wilke $\mathrm{H}$ : Treatment strategies in gastric cancer Dtsch Arztebl Int 108: 698-705, 2011.

5. Bang YJ, Van Cutsem E, Feyereislova A, et al: Trastuzumab in combination with chemotherapy versus chemotherapy alone for treatment of HER2-positive advanced gastric or gastro-oesophageal junction cancer (ToGA): a phase 3 , open-label, randomised controlled trial. Lancet 376: 687-697, 2010.

6. De Vita F, Giuliani F, Silvestris N, et al: Current status of targeted therapies in advanced gastric cancer. Expert Opin Ther Targets 16 (Suppl 2): S29-S34, 2012.

7. Naik S, Dothager RS, Marasa J,Lewis CL and Piwnica-Worms D: Vascular endothelial growth factor receptor-1 is synthetic lethal to aberrant beta-catenin activation in colon cancer. Clin Cancer Res 15: 7529-7537, 2009.

8. Tyner JW, Deininger MW, Loriaux MM, et al: RNAi screen for rapid therapeutic target identification in leukemia patients. Proc Natl Acad Sci USA 106: 8695-8700, 2009.

9. Giamas G, Filipovic A, Jacob J, et al: Kinome screening for regulators of the estrogen receptor identifies LMTK3 as a new therapeutic target in breast cancer. Nat Med 17: 715-719, 2011.

10. Stebbing J, Filipovic A, Ellis IO, et al: LMTK3 expression in breast cancer: association with tumor phenotype and clinical outcome. Breast Cancer Res Treat 132: 537-544, 2011.

11. Camargo MC, Goto Y, Zabaleta J, Morgan DR, Correa P and Rabkin CS: Sex hormones, hormonal interventions, and gastric cancer risk: a meta-analysis. Cancer Epidemiol Biomarkers Prev 21: 20-38, 2012.

12. Sheh A, Ge Z, Parry NM, et al: $17 \beta$-estradiol and tamoxifen prevent gastric cancer by modulating leukocyte recruitment and oncogenic pathways in Helicobacter pylori-infected INS-GAS male mice. Cancer Prev Res (Phila) 4: 1426-1435, 2011.
13. Ryu WS, Kim JH, Jang YJ, et al: Expression of estrogen receptors in gastric cancer and their clinical significance. J Surg Oncol 106: 456-461, 2012.

14. Xu CY, Guo JL, Jiang ZN, et al: Prognostic role of estrogen receptor alpha and estrogen receptor beta in gastric cancer. Ann Surg Oncol 17: 2503-2509, 2010.

15. Deng H, Huang X, Fan J, et al: A variant of estrogen receptor- $\alpha$, ER- $\alpha 36$ is expressed in human gastric cancer and is highly correlated with lymph node metastasis. Oncol Rep 24: 171-176, 2010.

16. Zhou J, Teng R, Xu C, et al: Overexpression of ER $\alpha$ inhibits proliferation and invasion of MKN28 gastric cancer cells by suppressing $\beta$-catenin. Oncol Rep 30: 1622-1630, 2013.

17. Cho JY: Molecular diagnosis for personalized target therapy in gastric cancer. J Gastric Cancer 13: 129-135, 2013.

18. Gravalos C and Jimeno A: HER2 in gastric cancer: a new prognostic factor and a novel therapeutic target. Ann Oncol 19: $1523-1529,2008$

19. Jorgensen JT and Hersom M: HER2 as a prognostic marker in gastric cancer - a systematic analysis of data from the literature. J Cancer 3: 137-144, 2012

20. Furukawa H, Iwanaga T, Koyama $H$ and Taniguchi H: Effect of sex hormones on the experimental induction of cancer in rat stomach - a preliminary study. Digestion 23: 151-155, 1982.

21. Lindblad M, Ye W, Rubio C and Lagergren J: Estrogen and risk of gastric cancer: a protective effect in a nationwide cohort study of patients with prostate cancer in Sweden. Cancer Epidemiol Biomarkers Prev 13: 2203-2207, 2004.

22. Chandanos E and Lagergren J: Oestrogen and the enigmatic male predominance of gastric cancer. Eur J Cancer 44: 2397-2403, 2008.

23. Gan L, He J, Zhang X, et al: Expression profile and prognostic role of sex hormone receptors in gastric cancer. BMC Cancer 12: 566, 2012.

24. Matsuyama S, Ohkura Y, Eguchi H, et al: Estrogen receptor beta is expressed in human stomach adenocarcinoma. J Cancer Res Clin Oncol 128: 319-324, 2002.

25. Wang M, Pan JY, Song GR, Chen HB, An LJ and Qu SX: Altered expression of estrogen receptor alpha and beta in advanced gastric adenocarcinoma: correlation with prothymosin alpha and clinicopathological parameters. Eur J Surg Oncol 33: 195-201, 2007.

26. Chandanos E, Rubio CA, Lindblad M, et al: Endogenous estrogen exposure in relation to distribution of histological type and estrogen receptors in gastric adenocarcinoma. Gastric Cancer 11: $168-174,2008$.

27. Kitaoka H: Chemo-endocrine therapy of diffuse carcinoma of the stomach and its clinical evaluation. Jpn J Cancer Clinics 31 (Suppl 9): 1189-1194, 1985 (In Japanese).

28. Harrison JD, Morris DL, Ellis IO, Jones JA and Jackson I: The effect of tamoxifen and estrogen receptor status on survival in gastric carcinoma. Cancer 64: 1007-1010, 1989.

29. Kojima $\mathrm{O}$ and Takahashi T: Endocrine therapy of scirrhous carcinoma of the stomach. Jpn J Cancer Chemother 13: 2526-2531, 1986 (In Japanese).

30. Kitaoka H: Sex hormone dependency in diffuse carcinoma of the stomach and results of chemo-endocrine therapy. Jpn J Cancer Clinics 30 (Suppl 6): 741-748, 1984 (In Japanese).

31. Kim MJ, Cho SI, Lee KO, Han HJ, Song TJ and Park SH: Effects of $17 \beta$-estradiol and estrogen receptor antagonists on the proliferation of gastric cancer cell lines. J Gastric Cancer 13: 172-178, 2013.

32. Shi H, Wu J, Ji M, et al: Serum lemur tyrosine kinase 3 expression in colorectal cancer patients predicts cancer progression and prognosis. Med Oncol 30: 754, 2013.

33. Wakatsuki T, LaBonte MJ, Bohanes PO, et al: Prognostic role of lemur tyrosine kinase-3 germline polymorphisms in adjuvant gastric cancer in Japan and the United States. Mol Cancer Ther 12: 2261-2272, 2013 